\title{
Studying The Influence of Various Geometrical Parameters of Single-Walled Carbon Nano-Tubes of Armchair Chirality Type on Its Mechanical Behavior
}

\author{
Hussein Zein ${ }^{1,2}$ \\ ${ }^{1}$ Mechanical Engineering Department, College of Engineering, Qassim University, Qassim, Saudi Arabia \\ ${ }^{2}$ Mechanical Design and Production Department, Faculty of Engineering, Cairo University, Giza, Egypt \\ Email address: \\ dr.husseinzein@qec.edu.sa
}

\section{To cite this article:}

Hussein Zein. Studying The Influence of Various Geometrical Parameters of Single-Walled Carbon Nano-Tubes of Armchair Chirality Type on Its Mechanical Behavior. World Journal of Applied Chemistry. Vol. 3, No. 1, 2018, pp. 17-27. doi: 10.11648/j.wjac.20180301.13

Received: October 21, 2017; Accepted: November 21, 2017; Published: January 10, 2018

\begin{abstract}
In the present work, the profound information on the mechanical properties of carbon nano-tubes (CNTs) were presented as an application of nano-technology in nano-materials are being most powerful in the current world. This would focuses/emphasis for designing and optimizing the CNTs based materials. Computational modeling technique was applied and developed to examine the mechanical characteristics for single-walled carbon nano-tube (SWCNT) of armchair chirality type. The atomistic based finite element method (FEM) was used to investigate the influence of various geometrical properties (diameter, wall thickness, and height-to-diameter ratio) of SWCNT armchair of chirality type on Poisson's ratio and Young's modulus values. Atomistic based finite element modeling was successfully developed and explored the mechanical behaviour of SWCNT exactly. The results were shown that the investigated geometrical parameters had much influenced on the mechanical properties of SWCNTs.
\end{abstract}

Keywords: Carbon Nano-Tube, Finite Element, Geometrical Parameters, Young's Modulus, Poisson's Ratio, Armchair

\section{Introduction}

Nanotubes are like a powder or dark ash. In general, the carbon nano-tube (CNT) is a truly wrapped up graphene sheets that set up empty strings owning dikes with single atom width [1]. The formation of carbon nanotube has three independent contact regions such as internal surface, external surface contact, and the contact between two ends. In fact, the nanotubes are being classified based on several parameters such as construction with one wall, construction with several walls as much as possible and the arrangement of carbon nano-tubes (CNTs) [1-5]. Moreover, both single wall nano-tube (SWNT) and multiple wall nanotubes (MWNTs) are being used as commercial products in numerous applications in day-to-day life [5] as the nanotubes products are having much flexibility [4]. Nanotubes can be used extensively in modern applications [6] such as micro and nano-electronics devices, reinforcement for composite materials, gas container, as a coating plate for radar, many capacitors, utilized in Atomic Force Microscope (AFM), technical textiles, biosensors for harmful gases, batteries with improved lifetime, additional durable fibers etc. [6]. Also, carbon nanotubes appeared as appropriate selection not only for treatment supply but also as a useful instrument in cancer diagnosis [7]. Smalley and Yacobson [8] have found the molecular arrangements of carbon in the 1980s. It was reported that carbon molecules were arranged within arbon atoms in the geometric forms of the sphereshaped crate. The carbon atoms in the crate are composed of hexagonal and pentagonal circles [9]. At the same time, Ijima [10] was presented about CNTs who characterized the CNTs as a moderately tall and thinning fullerene structures. It was found that carbon nano-tube walls were arranged in the form of hexagonal carbon cells with sphere-shaped heads at the ends. The binding of two heads together could form a fullerene [11].

CNTs have prodded extensive interest among researchers on account of their typical physical properties since 1991. As the name suggest, CNTs are having lengthy hollow nanostructures with walls formed by sheets of carbon. The CNTs are generally classified into two types namely; (i) single-walled nanotubes (SWNT) which is consisting of an 
atom of a thick layer of graphene $[12,13]$, and (ii) multiwalled nanotubes (MWNT) which (loosely) is a concentric tubes of graphene [13]. These nanostructures have possessed to own peculiar electronic and thermal properties; electric current capacity which exceed that of copper wires by 1000 times [14]; thermal conductivity greater than that of diamond [15] etc. In addition to exceptional electronic and thermal properties, CNTs have also shown remarkable mechanical properties which includes extremely higher in elastic modulus and strengths. Further, based on several researchers works, it was found that Young's modulus value of CNTs is having a higher value of more than $1 \mathrm{TPa}$ and ultimate tensile strength of greater than $150 \mathrm{GPa}$ that far away from the properties of high strength steels [16-18]. Due to these distinguished mechanical properties of CNT's, the science research communities have focused to explore unknown things from CNTs from past two decades. Several studies have presented about CNTs in the form of its atomic structure and its potential applications in physics, chemistry, materials science and engineering fields. CNT's are being used in various fields, which include reinforcements in composite materials, nano-fibers, catalysts, and numerous elements of novel electronic and thermal equipment [9, 19-24].

CNTs are one of potential candidate materials, which show many useful characteristics in the form of mechanical, thermal and electronics [25]. The major futuristic characters of CNT's are its chemical stability, high surface region, smallest dimensions, negligible mass, high stiffness and excellence in optical properties, electronic properties, and thermal behavior. These interesting properties of CNT's would motivate its applications into energy storages and nano-mechanical systems [9, 24, 26-28]. The modeling of CNT's is based on like construction of architecture in which the boundary conditions have to be given as constrained inputs and then simulation procedures are to be followed. In general, theoretical modeling of CNT behavior could be classified into three sets, namely, atomistic modeling, nanoscale continuum modeling and continuum modeling [29]. The simulation of molecular dynamics could be used to develop the elastic constants for SWCNT [30]. Further, the numeric values of Young's modulus, the modulus of rigidity and Poisson's ratio could be computed using the values of elastic constants [30]. The computations of material density as functional criteria could be used to examine the intrinsic mechanical properties of SWNT by the nano-tube diameter ranges from $0.3 \mathrm{~nm}$ to $0.8 \mathrm{~nm}$ [31]. By using this theory, it was found that the modulus of elasticity was exhibited distinguished value at the diameter less than $0.4 \mathrm{~nm}$ due to its higher value of the radius of curvature [31]. Ghadyani et al. [32] study the effect of the chiral angle and the aspect ratio on the modulus of rigidity for SWCNTs. This study is valuable for choosing the best configurations for SWCNTs.

Based on several researchers, the investigation towards the modeling of mechanical behavior of CNTs is still an open field for research. Therefore, in the present research work, systematic feasible simulation procedure was developed to investigate the modulus of elasticity for SWCNT in armchair chirality type. Further, finite element simulation (FES) was also developed to examine the effect of dimensional parameters of SWCNT armchair chirality type on its modulus of elasticity. For this, the various geometrical parameters such as diameter, wall thickness, and height-to-diameter ratio were taken for investigation.

\section{Finite Element Model (FEM)}

Based on the main objective of the present work, the finite element based simulation procedure was developed to predict the mechanical properties for SWCNT using atomistic modeling technique. For this, the following steps are to be used:

(1) Generate the structural model of SWCNT using Nanotube Modeler software; shown in Figure 1.

(2) Export the model generated in step (1) as a PDB file; presented in Figure 2.

(3) Write a Python code by using the Py Charm program to convert the PDB file into Ansys nodes, key points, and lines; displayed in Figure 3.

(4) The output text file in step (3) would be in the XYZ directory, as created in (1).

(5) Then imported the text file in Ansys program for tensile test simulation.

(6) Then export the text file from Ansys program contains the reaction forces and the extensions values which result from the simulation of the tensile test for further analysis; presented in Figure 4.
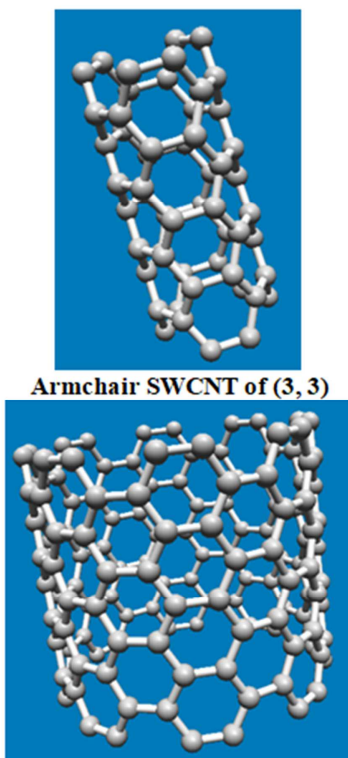

Armchair SWCNT of $(8,8)$
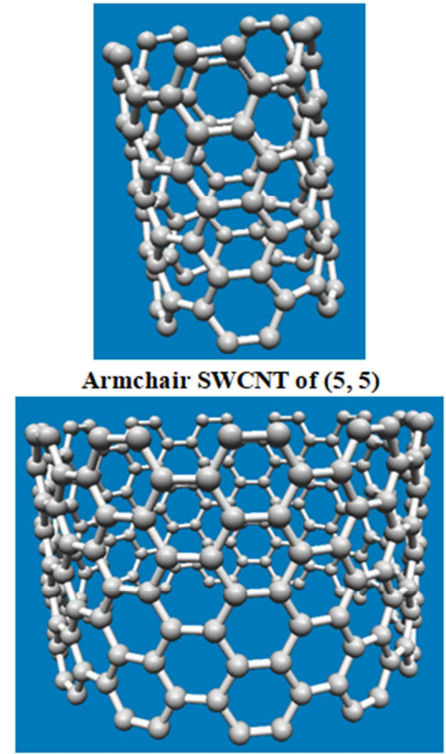

Armchair SWCNT of $(12,12)$
Figure 1. Generation of the structural models of SWCNT using Nanotube Modeler with height of $9.769 \mathrm{~nm}$.

Some important points are to be taken into account to create a finite element model for an SWCNT. These points are: (i) understanding the geometry of nano-tubes; (ii) determining the atomic coordinates for nano-tube formation, (iii) the base of atomic modeling which mainly depends on 
atomic coordinates, (iv) molecular bonding between atoms which should be built up as per experimental observations, and (v) chemical bond should be substituted by a suitable simulation element called beam element.

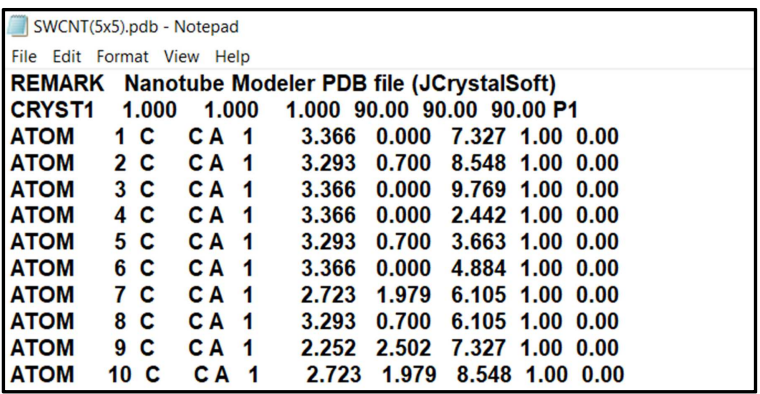

Figure 2. Export the model generated in Nanotube Modeler software as a $P D B$ file.
The BEAM188 element in finite element analysis (FEA) program using ANSYS was used to emulate the carbon chemical bonds. It is to be noted here that the crosssectional area of the BEAM188 element is supposed to be a circular and uniform. The diameter (d), modulus of elasticity (E) and modulus of rigidity $(\mathrm{G})$ are the major input parameters of the BEAM188 element. These parameters are to be calculated using the following equations [33-37]:

$$
\begin{aligned}
& \mathrm{d}=\sqrt[4]{\frac{K_{\theta}}{K_{r}}} \\
& \mathrm{E}=\frac{L K_{r}^{2}}{4 \pi K_{\theta}} \\
& \mathrm{G}=\frac{L K_{r}^{2} K_{\tau}}{8 \pi K_{\theta}^{2}}
\end{aligned}
$$

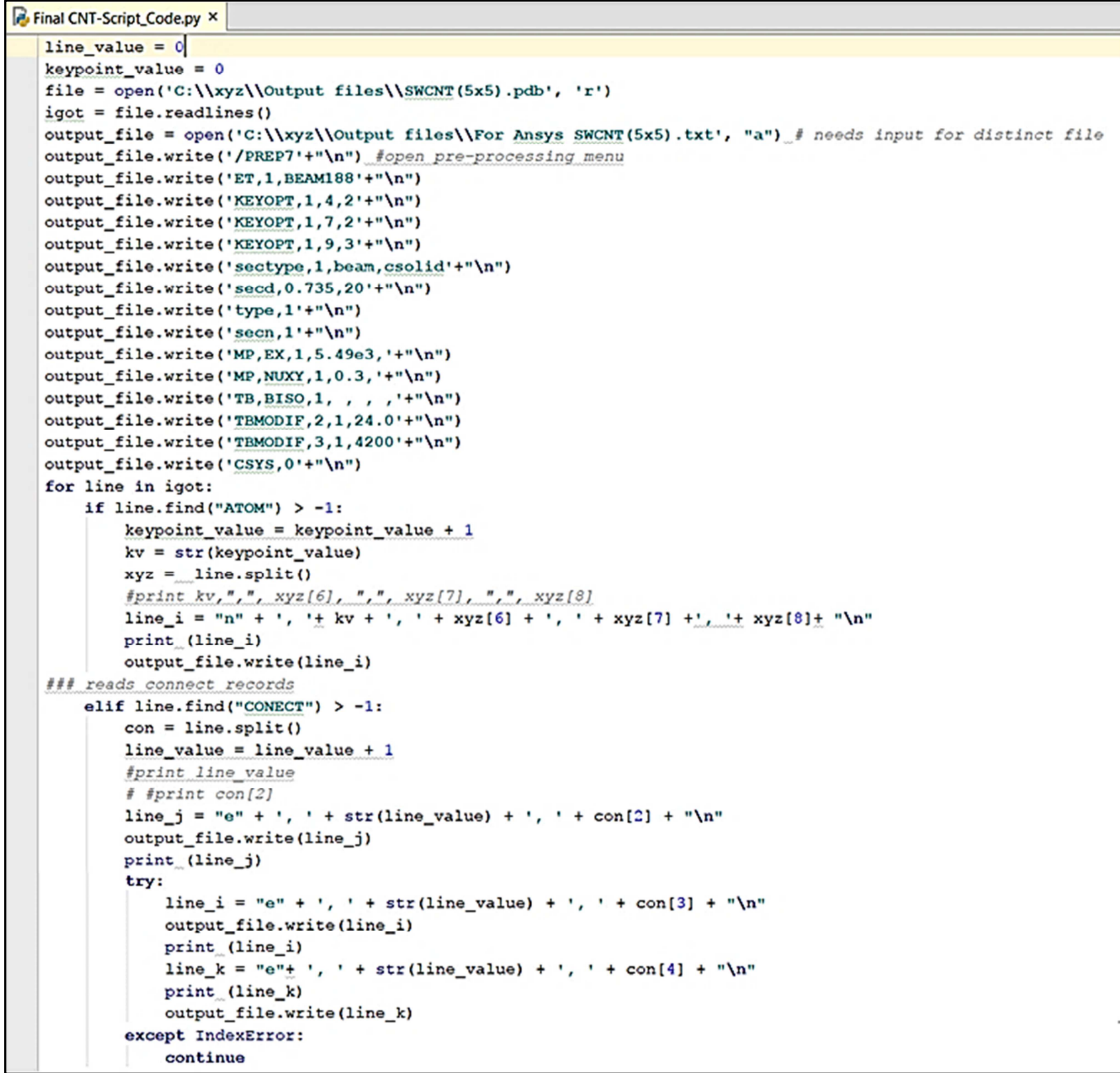

Figure 3. Write a Python code by using Py Charm program to convert the PDB file into Ansys nodes, key points, and lines. 
All the above parameters were obtained from [38-39], where:

$\mathrm{K}_{\theta}, \mathrm{K}_{\mathrm{r}}$, and $\mathrm{K}_{\tau}=$ are the molecular mechanic's parameters, and

$\mathrm{L}=\mathrm{a}_{\mathrm{c}-\mathrm{c}}=$ is the length of $\mathrm{C}-\mathrm{C}$ bond in carbon nano-tube.

$\mathrm{K}_{\theta}=126 \mathrm{kcal} \mathrm{mole} \mathrm{rad}^{-2}=0.8758 \mathrm{nN} \mathrm{nm} / \mathrm{rad}^{2}$

$\mathrm{K}_{\mathrm{r}}=938 \mathrm{kcal} \mathrm{mole}^{-1} \AA^{-2}=651.97 \mathrm{nN} / \mathrm{nm}$

$\mathrm{K}_{\tau}=40 \mathrm{kcal} \mathrm{mole} \mathrm{rad}^{-2}=0.278 \mathrm{nN} \mathrm{nm} / \mathrm{rad}^{2}$

$\mathrm{L}=0.142 \mathrm{~nm}$

\begin{tabular}{|c|c|}
\hline \multicolumn{2}{|c|}{ OutputTXT - Notepad } \\
File Edit Format View Help \\
-193.561 & 0.200 \\
-374.769 & 0.400 \\
-555.173 & 0.600 \\
-733.980 & 0.800 \\
-910.575 & 1.000 \\
-1084.403 & 1.200 \\
-1255.025 & 1.400 \\
-1422.049 & 1.600 \\
-1585.162 & 1.800 \\
-1744.112 & 2.000 \\
-1898.710 & 2.200 \\
-2048.817 & 2.400 \\
-2194.340 & 2.600 \\
-2335.229 & 2.800 \\
-2471.466 & 3.000 \\
-2603.067 & 3.200 \\
-2730.069 & 3.400 \\
-2852.531 & 3.600 \\
-2970.531 & 3.800 \\
-3084.157 & 4.000 \\
-3193.511 & 4.200 \\
-3298.702 & 4.400 \\
-3399.844 & 4.600 \\
-3497.056 & 4.800 \\
-3590.461 & 5.000 \\
-3680.181 & 5.200 \\
-3766.338 & 5.400 \\
-3849.053 & 5.600 \\
-3928.447 & 5.800 \\
-4004.638 & 6.000 \\
-4077.743 & 6.200 \\
-4147.877 & 6.400 \\
-4215.148 & 6.600 \\
-4279.666 & 6.800 \\
-4341.534 & 7.000 \\
-4400.852 & 7.200 \\
-4457.718 & 7.400 \\
-4512.225 & 7.600 \\
-4564.465 & 7.800 \\
-4614.523 & 8.000 \\
& \\
& \\
\hline
\end{tabular}

Figure 4. Export the text file from Ansys program contains the reaction forces and the extensions values.

Then, the required input parameters of the BEAM 188 element were calculated by the following: the diameter of the BEAM188 element (d) is $0.147 \mathrm{~nm}$, Young's modulus (E) is $5.49 \mathrm{TPa}$ and the modulus of rigidity $(\mathrm{G})$ is $0.871 \mathrm{TPa}$. Using the isotropic elasticity concept, the equation of $[\mathrm{G}=\mathrm{E} / 2$ $(1+v)$ ] was used to determine the value of Poisson's ratio $(v)$ in which the values of $E$ and $G$ are to be used. However, the observed result of Poisson's ratio $(v)$ using this equation was 2.15 which is larger than 0.5 which violate the isotropic elasticity principle. Therefore, Natsuki et al. [40] proposed a new procedure of numerical estimation of Poisson's ratio $(v)$ for the BEAM188 element. These researchers were calculated the Poisson's ratio $(v)$ value to be around 0.27 by using the following equation:

$$
v=\frac{\mathrm{L}^{2} \mathrm{~K}_{\mathrm{r}}-6 \mathrm{~K}_{\Theta}}{\mathrm{L}^{2} \mathrm{~K}_{\mathrm{r}}+18 \mathrm{~K}_{\Theta}}
$$

Finally, the yield strength of the BEAM188 element is 47.22 GPa according to reference [41]. Figure 5 displays the finite element analysis model for single-walled carbon nanotube (SWCNT) armchair chirality type.

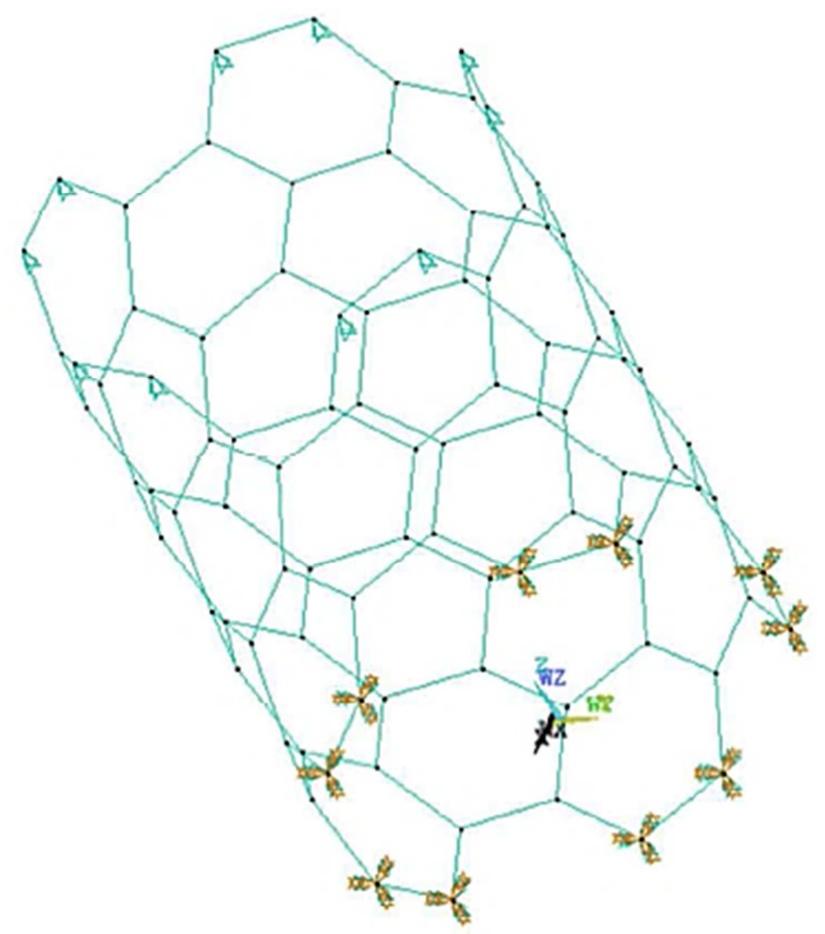

Figure 5. The finite element analysis model for SWCNT.

\section{Results and Discussion}

In this research, the mechanical properties of single-walled carbon nano-tube were predicted by utilizing the concept of the finite element model based simulation procedure using tensile test results of SWCNT. The nano-tube was modeled like a hexagonal grid of bonds. Single-walled nano-tube with different geometrical parameters and chirality for armchair type were modeled with bonds between the atoms which considered as a beam element. In this research, a finite element simulation (FES) was carried out to investigate the influence of geometrical parameters (diameter, thickness, and height to diameter ratio) on the modulus of elasticity (E) and the Poisson's ratio (v) for SWCNT armchair nano-tube type. To estimate the tensile stress and the tensile strain from the FE results, the following equations were utilized:

$$
\begin{aligned}
& \sigma=\frac{\mathrm{F}_{\mathrm{z}}}{\mathrm{A}} \\
& \varepsilon_{h}=\frac{\Delta \mathrm{h}}{\mathrm{h}_{\mathrm{t}}}
\end{aligned}
$$


Where:

$\sigma=$ the tensile stress,

$\mathrm{F}_{\mathrm{z}}=$ is the tensile force applied at one end of the nano-tube during the tensile test,

$\mathrm{A}=$ is the nano-tube cross-section area, which is:

$$
\mathrm{A}=\pi \cdot \mathrm{d}_{\mathrm{t} .} \mathrm{t}
$$

(Where: $d_{t}=$ is the diameter of nano-tube, and $t=$ nanotube wall thickness),

$\varepsilon_{\mathrm{h}}=$ the tensile strain,

$\Delta \mathrm{h}=$ is the extension during the tensile test, and

$\mathrm{h}_{\mathrm{t}}=$ is the interlayer original nano-tube height.

To calculate the axial modulus of elasticity (E), the following equation was utilized in elastic zone:

$$
\left.E=\frac{\sigma}{\varepsilon_{l}}\right)_{\text {Elastic zone }}
$$

Also, the finite element results were utilized to compute the Poisson's ratio ( $v$ ) for single-walled carbon nano-tube. To evaluate the Poisson's ratio ( $v$ ) from the FE results, the following equations were utilized:

$$
\begin{aligned}
& v=\frac{-\varepsilon_{r}}{\varepsilon_{h}} \\
& \varepsilon_{r}=\frac{\Delta r_{t}}{r_{t}}
\end{aligned}
$$

Where:

$v=$ is the Poisson's ratio,

$\varepsilon_{\mathrm{r}}=$ is the radial strain,

$\Delta \mathrm{r}_{\mathrm{t}}=$ is the change in the nano-tube radius, and

$\mathrm{r}_{\mathrm{t}}=$ is the nano-tube radius.

The estimated modulus of elasticity was $1.2 \mathrm{TPa}$ at a nanotube wall thickness of $0.34 \mathrm{~nm}$ in the case of SWCNT armchair chirality type, which reached well agreement with other values of literature presented in the following table (Table 1). Furthermore, the modulus of elasticity of the present FEA work $(1.2 \mathrm{TPa})$ is even closer to the average

\begin{tabular}{|c|c|c|c|c|}
\hline Researchers & Method & $\begin{array}{l}\text { CNT wall thickness } \\
(\mathrm{t}, \mathrm{nm})\end{array}$ & $\begin{array}{l}\text { Chirality } \\
(\mathbf{n}, \mathbf{n})\end{array}$ & $\begin{array}{l}\text { Modulus of } \\
\text { Elasticity (E, TPa) }\end{array}$ \\
\hline The Present FEM work & Atomistic modeling technique & 0.34 & $(5,5)$ & 1.2 \\
\hline Giannopoulos et al. [42] & Atomistic modeling technique & 0.34 & $(5,5)$ & 1.2850 \\
\hline Lier et al. [43] & ab initio multiplicative integral approach (MIA) & 0.34 & $(5,5)$ & 1.06 \\
\hline Shokrieh and Rafiee [44] & Nano-scale continuum mechanics & 0.34 & $(5,5)$ & 1.033 \\
\hline $\mathrm{Lu}[45]$ & Molecular dynamics & 0.34 & $(5,5)$ & 0.971 \\
\hline Jin and Yuan [46] & Molecular dynamics & 0.34 & $(6,6)$ & 1.217 \\
\hline
\end{tabular}
modulus of elasticity of the experimental work (1.002 TPa) as mentioned in the literature [26].

Table 1. Comparison of modulus of elasticity between the present work and other values of literature.

\subsection{Effect of Variation of Carbon Nano-Tube Diameter $\left(d_{t}\right)$}

Carbon nano-tube diameter was computed by using the following equation [47]:

$$
d_{t}=\frac{a_{0}}{\pi} \sqrt{3\left(n^{2}+m^{2}+n m\right)}
$$

Where:

$\mathrm{d}_{\mathrm{t}}=$ nano-tube diameter,

$\mathrm{a}_{\mathrm{o}}=$ Length of unit vector, $\mathrm{a}_{\mathrm{o}}=\sqrt{3} \mathrm{a}_{\mathrm{c}-\mathrm{c}}$

(Where: $\mathrm{a}_{\mathrm{c}-\mathrm{c}}=0.142 \mathrm{~nm}=$ the length of the C-C bond), and $\mathrm{n}, \mathrm{m}=$ are chirality index of nano-tnbes.

Figure 6 shows the engineering stress - engineering strain curves at different values of carbon nano-tube diameter for single-walled carbon nano-tube armchair type. It was observed the interesting results that when the geometrical properties of CNT's started to decreases the observed stress value increases drastically for the same amount of strain. SWCNT of $12 \times 12$ chirality type had exhibited maximum stress value of $235 \mathrm{GPa}$ at 0.8 fraction strain. Whereas, SWCNT of $3 \times 3$ chirality type had produced $1125 \mathrm{GPa}$ at the same amount of 0.8 fraction strain which was 4.8 times higher than that of $12 \times 12$ chirality type SWCNT. This fact in real case SWCNT was attributed to more surface energy usually available with the function of decreasing its geometrical properties that enhance the strength of nanomaterials. The same phenomenological behavior was observed by simulation results which meant it correlates with the experimental and materials behavior. Therefore, the present work of developed atomistic model would predict the stress-strain behavior of SWCNT consequently it eliminates the experimental work and its cost. Further, the increasing stress value with a function of decreasing the geometrical properties in simulation point of view was attributed to more value of the radius of curvature of SWCNT.

The variation of modulus of elasticity (E) with the function of the diameter of SWCNT $\left(\mathrm{d}_{\mathrm{t}}\right)$ is shown in Figure 7. It is to be noted here that the observed modulus of elasticity (E) value was $2 \mathrm{TPa}, 1.23 \mathrm{TPa}, 0.8 \mathrm{TPa}$ and $0.5 \mathrm{TPa}$ for CNT diameter of $4 \mathrm{~nm}, 6.5 \mathrm{~nm}, 10.75 \mathrm{~nm}$ and $16.25 \mathrm{~nm}$ respectively. These results were explained that the predicted modulus of elasticity by atomistic FE model for CNT of 4 $\mathrm{nm}$ had $400 \%$ higher than CNT of $16.25 \mathrm{~nm}$ which exactly matches with experimental analysis of modulus of elasticity with the function of decreasing of its diameter [25, 26].

The variation of Poisson's ratio $(v)$ with the function of CNT diameter is also shown in Figure 8 in which the predicted Poisson's ratio was started to decreases with increasing the CNT diameter. In general, a higher value of Poisson's ratio in the material would exhibit more stress (Figure 6) and hence the smallest diameter of CNT's investigated here had produced more Poisson's ratio (Figure 8) and stress values (Figure 6). Based on these results, it can be understood that the developed atomistic modeling technique using the present finite element simulation can be used to predict the CNT's mechanical behavior exactly when compared to other's work. 


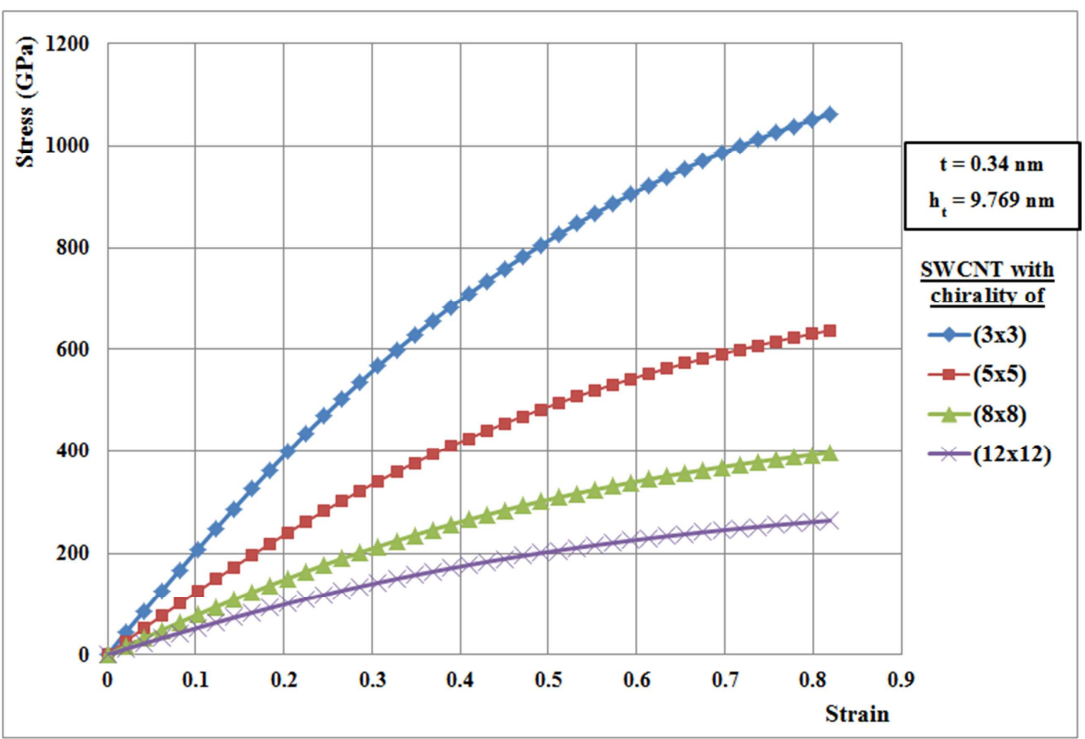

Figure 6. Engineering Stress - Engineering Strain curve for armchair carbon nano-tube type at different values of the CNT diameter $\left(d_{t}\right)$ for armchair chirality type.

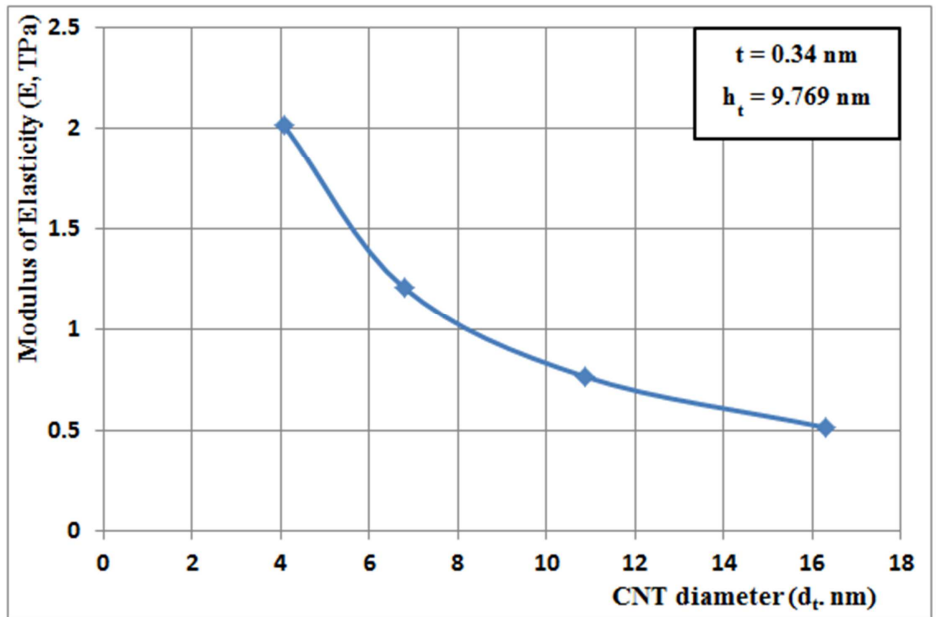

Figure 7. Variation of the modulus of elasticity $(E)$ with diverse values of the CNT diameter $\left(d_{t}\right)$ for armchair chirality type.

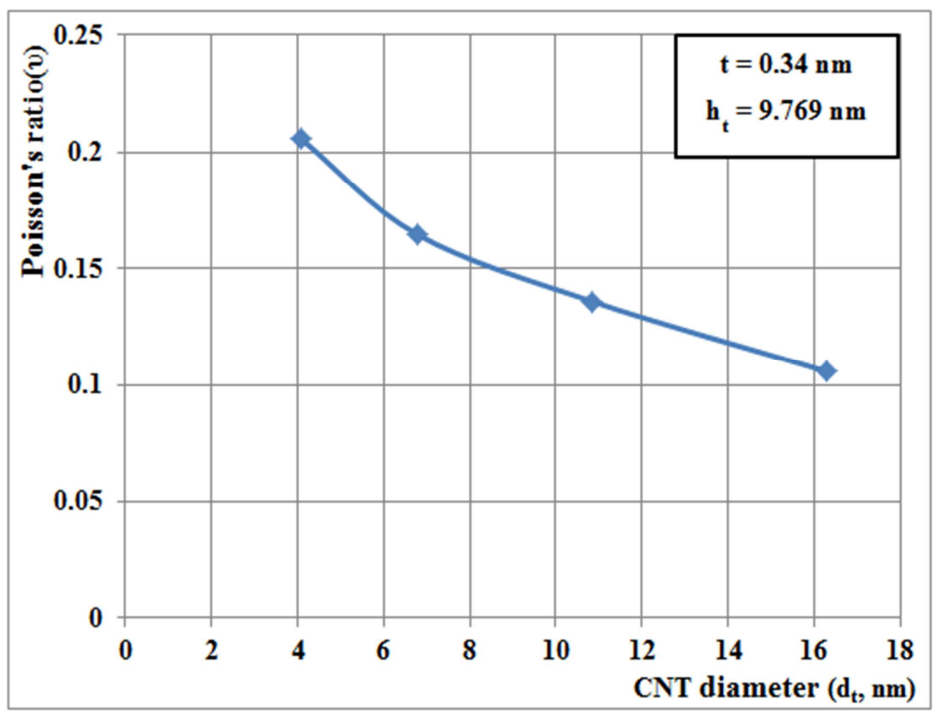

Figure 8. Change of the Poisson's ratio (v) at various values of carbon nano-tube diameter $\left(d_{t}\right)$ for armchair chirality type. 


\subsection{Effect of Variation of Carbon Nano-Tube Wall Thickness (t)}

The changing of the thickness of SWCNT in several ways was investigated by more researchers [48-51]. The nano-tube wall thickness of $0.066 \mathrm{~nm}$ was used by Mohammed Pour, 2013 [48] when investigating the numerical and experimental work of CNT's based polymeric composites using non-linear finite element method. Further, several researchers have used $0.34 \mathrm{~nm}$ as the thickness of CNT's during its simulation [49-51].

The variation of engineering stress-engineering strain as a function of SWCNT thickness is shown in Figure 9. It is to be noted here that the simulated stress behavior was started to increases drastically when the thickness of SWCNT decreases from $0.69 \mathrm{~nm}$ to $0.066 \mathrm{~nm}$. For instance, the thickness of $0.69 \mathrm{~nm}$ SWCNT had exhibited the maximum stress value of $375 \mathrm{GPa}$ whereas $0.066 \mathrm{~nm}$ thickness of
SWCNT had produced the maximum stress value of 3250 GPa which was around $870 \%$ higher than the initial one. Further, it can be observed that there was a slight increase in maximum stress value when the thickness of SWCNT decreases from 0.69 to $0.34 \mathrm{~nm}$, it was increased considerably up to $0.147 \mathrm{~nm}$ thickness of SWCNT and then it increased drastically at $0.066 \mathrm{~nm}$ thickness of SWCNT.

The variation of modulus of elasticity of SWCNT with the function of its thickness is shown in Figure 10. These results were explained that the modulus of elasticity of SWCNT was started to increases when the thickness of SWCNT decreases. The wall thickness of $0.066 \mathrm{~nm}$ SWCNT had shown the modulus of elasticity value of $6.2 \mathrm{TPa}$ whereas $0.69 \mathrm{~nm}$ wall thickness of SWCNT had produced only $0.62 \mathrm{TPa}$ which meant around 10 times more in a lower value of SWCNT thickness.

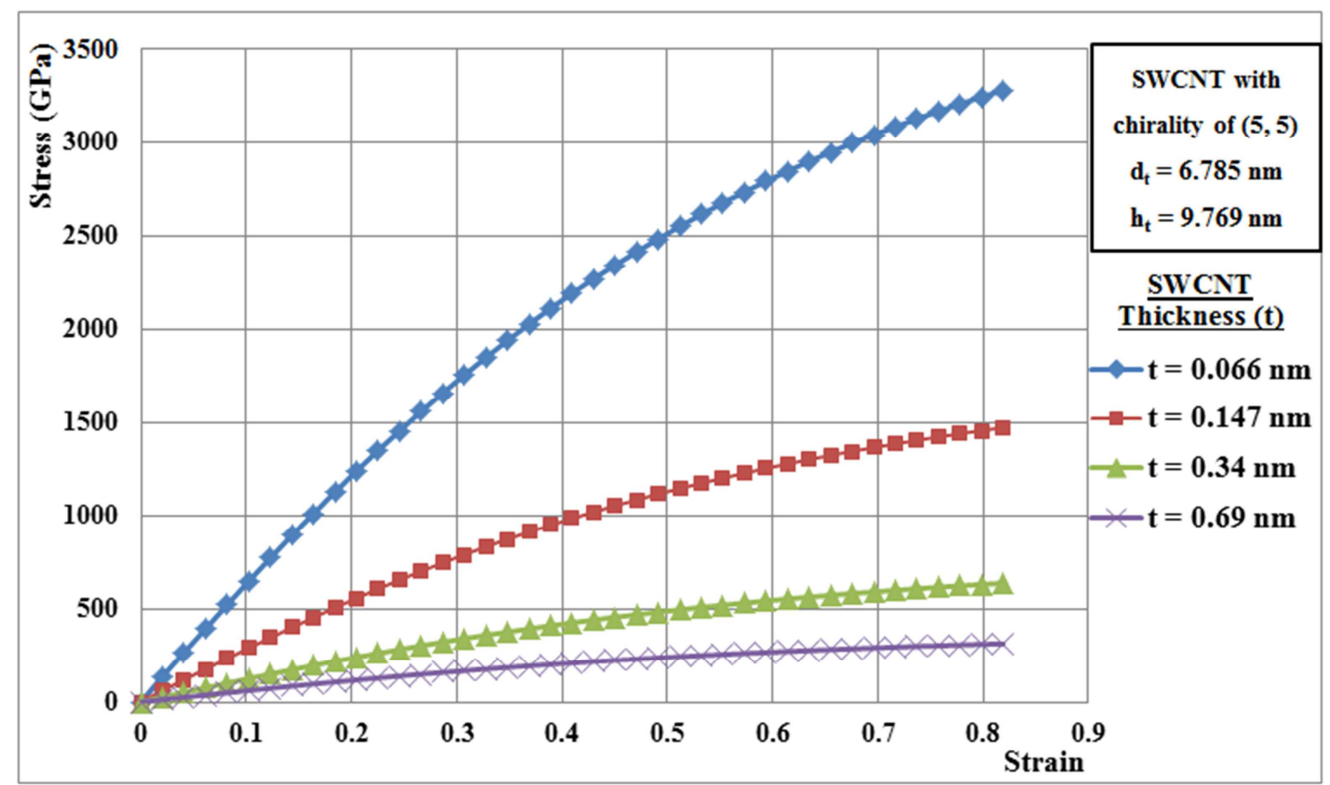

Figure 9. Engineering Stress - Engineering Strain curve for armchair carbon nano-tube type at diverse values of the CNT wall thickness (t) for armchair chirality type.

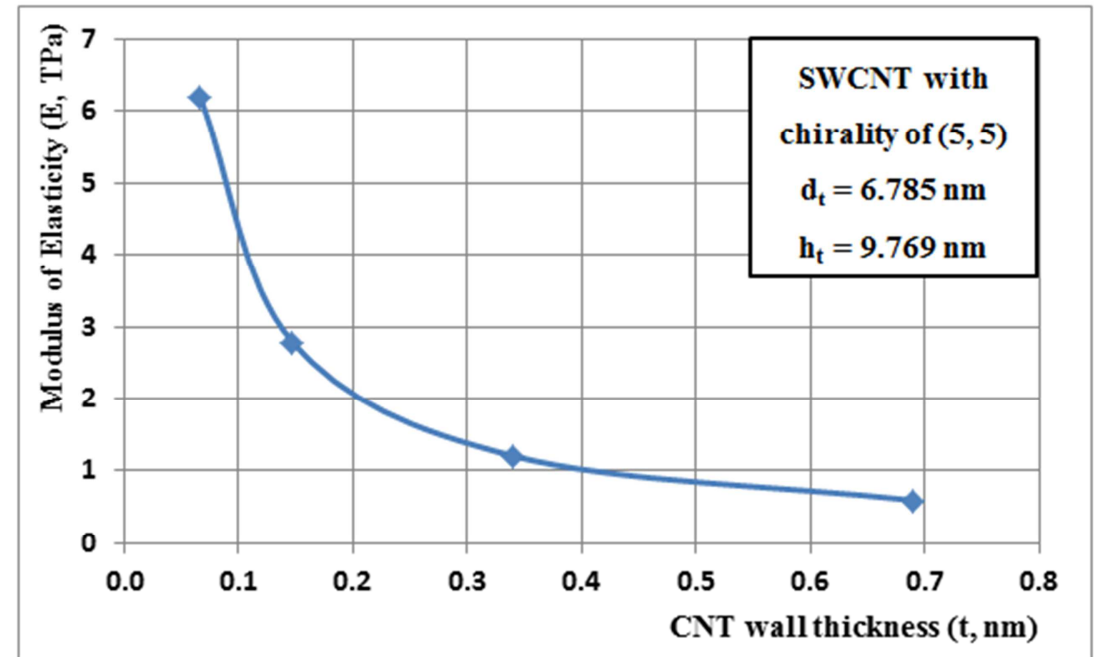

Figure 10. Variation on the modulus of elasticity $(E)$ with different values of the CNT wall thickness (t) for armchair chirality type. 


\subsection{Effect of Variation of Carbon Nano-Tube Height-to-Diameter Ratio $\left(h_{t} / d_{t}\right)$}

The effects of SWCNT geometry of its diameter and its thickness on its mechanical behavior were discussed early. In a similar manner, the SWCNT height-to-diameter ratio $\left(\mathrm{h}_{\mathrm{t}} / \mathrm{d}_{\mathrm{t}}\right)$ would also affect the mechanical behavior which was discussed in this section.

Figure 11 shows the variation of engineering stressengineering strain as a function of the height-to-diameter ratio of SWCNT. It can be noted here that as the height-todiameter ratio of SWCNT was started to increases, the observed value of stress also started to increases considerably. When the height-to-diameter ratio of SWCNT increases seven times with original, there was not that much improvement in engineering stress-strain behavior. However, as the height-to-diameter ratio of SWCNT increases fourteen times with initial, it had exhibited enhanced stress-strain behavior which is an interesting and these results would emphasis more on further research.

In a similar manner, the variation of modulus of elasticity (E) and Poisson's ratio with the function of the height-todiameter ratio are shown in Figure 12 and Figure 13 respectively. As the height-to-diameter ratio was increased, both of these values started to increases slightly and then it increased marginally which indicated the significance of height-to-diameter ratio geometry. Based on these results and finding, it can be concluded that the present atomistic finite element procedure would help us to design a proper SWCNT that can be used in real-time applications.

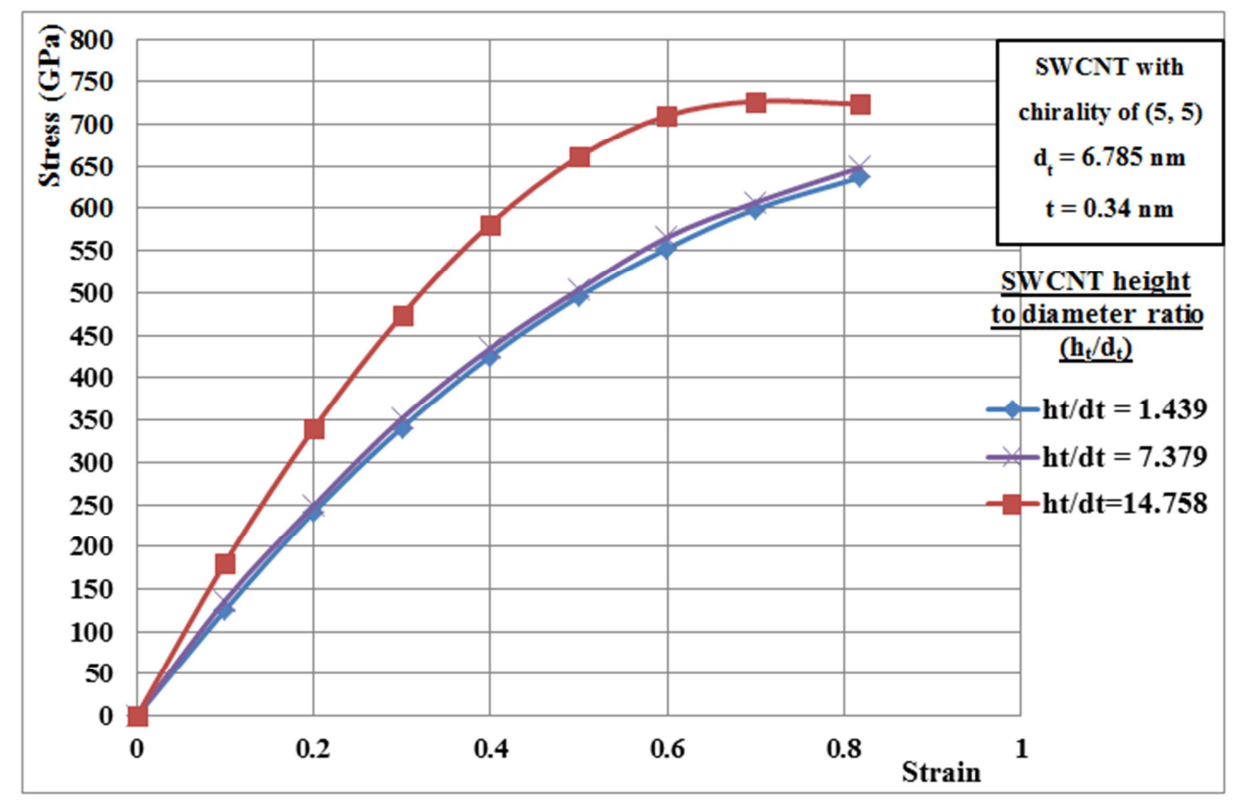

Figure 11. Engineering Stress - Engineering Strain curve for armchair carbon nano-tube type at varied values of the CNT height-to-diameter ratio $\left(h_{t} / d_{t}\right)$.

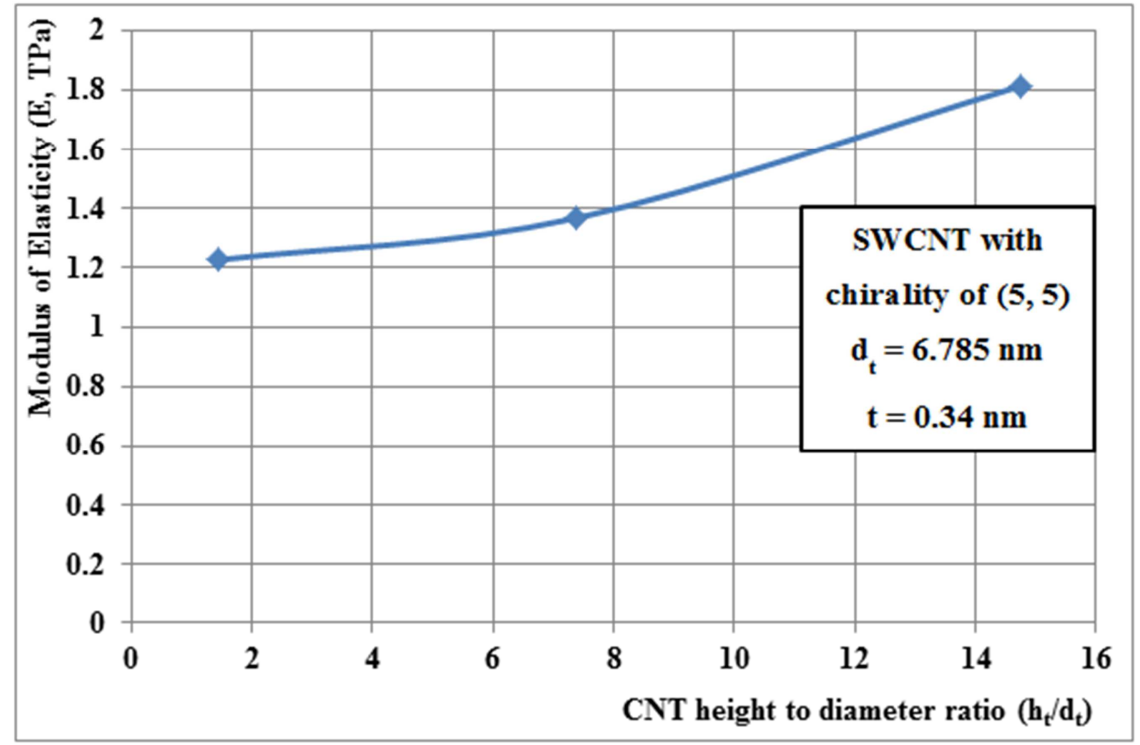

Figure 12. Variation of the modulus of elasticity (E) with different values of the CNT height-to-diameter ratio $\left(h_{t} / d_{t}\right)$ for armchair chirality type. 


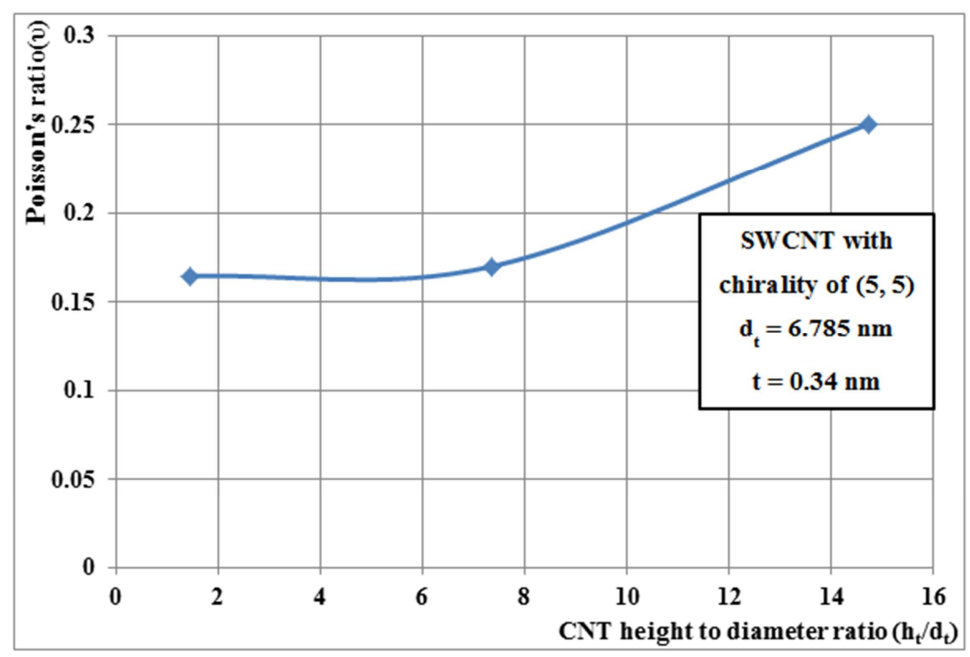

Figure 13. Change of the Poisson's ratio (v) with dissimilar values of the CNT height-to-diameter ratio $\left(h_{t} / d_{1}\right)$ for armchair chirality type.

\section{Conclusion}

Atomistic based finite element modeling technique was developed to predict the mechanical behavior of SWCNT of armchair chirality type in which various geometrical features, namely, CNT diameter, thickness and height-to-diameter ratio had been varied and achieved the interesting results. The following conclusions were drawn through the present investigation:

1. Novel atomistic modeling technique was developed using finite element analysis.

2. The simulated modulus of elasticity of $4 \mathrm{~nm}$ diameter SWCNT had exhibited $2 \mathrm{TPa}$ which was 4 times higher than $16.5 \mathrm{~nm}$ diameter SWCNT.

3. The smallest geometric properties of $3 \times 3$ SWCNT had produced the ultimate stress value of $1125 \mathrm{GPa}$ whereas $12 \times 12$ SWCNT had shown the ultimate stress value of $235 \mathrm{GPa}$ which was around 4.8 times lower than $3 \times 3$ SWCNT geometry for the same 0.8 fraction of strain.

4. Further, $0.34 \mathrm{~nm}$ thickness SWCNT had given the ultimate stress of $3250 \mathrm{GPa}$ which was $767 \%$ higher than $0.69 \mathrm{~nm}$ thickness SWCNT which implied clearly the significance of CNT thickness on its mechanical behavior.

5. In addition, the value of the height-to-diameter ratio of SWCNT of $1.44,7.38$ and 14.76 on its Poisson's ratio was $0.16,0.17$ and 0.25 respectively which emphasized that there was not that much significance in the value of Poisson's ratio up to 7.38. However, a higher value of 0.25 Poisson's ratio was obtained in the case of 14.76 height-to-diameter ratio of SWCNT.

6. Through this investigation, the scientific research community can design and develop an appropriate geometry of SWCNT for its targeted applications.

\section{References}

[1] Harris, P. J. F., Carbon Nanotubes and Related Structures, 1st ed., Cambridge University Press: Cambridge, 2001.
[2] Yan C., Liu J., Liu F., Wu J., Gao K., and Xue D. (2008). Tube Formation in Nanoscale Materials. Nanoscale Res. Lett. 3 (12), 473-480.

[3] Kuchibhatla, S. V., Karakoti, A. S., Bera, D., and Seal S. (2007). One Dimensional Nanostructured Materials. Prog. Mater Sci. 52 (5), pp. 699-913.

[4] Yang, P., The Chemistry of Nanostructured Materials, 1st ed., vol. 2, World Scientific: Singapore, 2011.

[5] Koch, C. C., Nanostructured Materials: Processing, Properties, and Applications, William Andrew: Norwich, 2006.

[6] Coombs, R. R. H., and Robinsons, D. W., Nanotechnology in Medicine and Biosciences, 1st ed., Gordon and Breach: New York, 1996.

[7] Sanginario, A., Miccoli, B., and Demarchi, D. (2017). Carbon Nanotubes as an Effective Opportunity for Cancer Diagnosis and Treatment. Biosensors 7, 9, 1-23.

[8] Smalley R. E., and Yakobson, B. I. (1998). The Future of the Fullerenes. Solid State Commun. 107, 597-606.

[9] Wong, E. W., Sheehan, P. E., and Lieber, C. M. (1997). Nanobeam Mechanics: Elasticity, Strength, and Toughness of Nanorods and Nanotubes. Science 277, 1971-1975.

[10] Iijima, S. (1991). Helical Microtubules of Graphite Carbon. Nature 354, 56-58.

[11] Spitalsky, Z., Tasis, D., Papagelis, K., and Galiotis, C. (2010). Carbon Nanotube Polymer Composites: Chemistry, Processing, Mechanical, and Electrical Properties. Prog. Polym. Sci. 35, 357-401.

[12] Bethune, D. S., Kiang, C. H., Devries, M. S., Gorman, G., Savoy, R., Vazquez, J., and Beyers, R. (1993). CobaltCatalyzed Growth of Carbon Nanotubes with Single-AtomicLayer Walls. Nature 363, 605-607.

[13] Coleman, J. N., Khan, U., Blau, W. J., and Gun'ko, Y. K. (2006). Small but Strong: a Review of the Mechanical Properties of Carbon Nanotube Polymer Composites. Carbon 44, 1624-1652.

[14] Collins, P. G., and Avouris, P. (2000). Nanotubes for Electronics. Scientific American 283, $62-69$. 
[15] Thostenson, E. T., Ren, Z., and Chou T. W. (2001). Advances in the Science and Technology of Carbon Nanotubes and their Composites: a Review. Compos. Sci. Technol. 61, 1899-1912.

[16] Zhang, X. F., Zhang, X. B., Tendeloo, G. V., Amelinckx, S., Beeck, M., and Landuyt, J. V. (1993). Carbon Nanotubes: their Formation Process and Observation by Electron Microscopy. J. Cryst. Growth 130, 368-382.

[17] Pipes, R. B., and Hubert, P. (2002). Helical Carbon Nanotube Arrays: Mechanical Properties, Compos. Sci. Technol. 62, 419-428.

[18] Salvetat, D. J. P., and Rubio, A. (2002). Mechanical Properties of Carbon Nanotubes: a Fiber Digest for Beginners. Carbon $40,1729-1734$.

[19] Thostenson, E. T., and Chou, T. W. (2002). Aligned Multi-Walled Carbon Nanotube Reinforced Composites: Processing and Mechanical Characterization. J. Phys. D Appl. Phys. 35, 77-80.

[20] Treacy, M. M. J., Ebbesen, T. W., and Gibson, J. M. (1996). Exceptionally High Young's Modulus Observed for Individual Carbon Nanotubes. Nature 381, 678-680.

[21] Salvetat, J. P. B., Briggs, G. A. D., Bonard, J. M., Bacsa, R. R., Kulik, A. J., Stöckli, T., Burnham, N. A., and Forró, L. (1999). Elastic and Shear Moduli of Single Walled Carbon Nanotube Ropes. Phys. Rev. Lett. 82, 944-947.

[22] Walters, D. A., Ericson, L. M., Casavant, M. J., Liu, J., Colbert, D. T., and Smith, K. A. et al. (1999). Elastic Strain of Freely Suspended Single Wall Carbon Nanotube Ropes. Appl. Phys. Lett. 74, 3803-3805.

[23] Yakobson, B. I., and Avouris, P. (2001). Mechanical Properties of Carbon Nanotubes. Topics Appl. Phys 80, 287-327.

[24] Yu, M. F., Lourie, O., Dyer, M. J., Moloni, K., Kelly, T. F., and Ruoff, R. S. (2000). Strength and Breaking Mechanism of Multiwalled Carbon Nanotubes Under Tensile Load. Science 287, 637-640.

[25] Li, W., Xie, S., Pan, Z., Chang, B., and Sun, L. (2000). Mechanical and Physical Properties on Carbon Nanotube. J. Phys. Chem. Solids 61, 1153-1158.

[26] Yu, M. F., Files, B. S., Arepalli, S., and Ruoff, R. S. (2000). Tensile Loading of Ropes of Single Wall Carbon Nanotubes and their Mechanical Properties. Phys. Rev. Lett. 84, 55525555 .

[27] Thostensona, E. T., Renb, Z., and Choua, T. W. (2001). Advances in the Science and Technology of Carbon Nanotubes and their Composites a Review. Compos. Sci. Technol. 61, 1899-1912.

[28] Dresselhaus, M. S., Dresselhaus, G., and Saito, R. (1995). Physics of carbon nanotubes. Carbon 33, 883-891.

[29] Rafiee, R., and Moghadam, R. (2014). On the Modeling of Carbon Nanotubes: A Critical Review. Journal of Composites: Part B 56, 435-449.

[30] Zaeri, M. M., Ziaei-Rad, S., and Shahidi, A. R. (2015). On the Elastic Constants of Single Walled Carbon Nanotubes. Procedia Materials Science 11, 666671.

[31] Hung, N. T., Truong, D. V., Thanh, V. V., and Saito, R. (2016). Intrinsic Strength and Failure Behaviors of Ultra-Small Single-Walled Carbon Nanotubes. Computational Materials Science 114, 167-171.
[32] Ghadyani, G., Soufeiani, L., and Öchsner, A. (2017). Angle Dependence of the Shear Behaviour of Asymmetric Carbon Nanotubes. Materials and Design 116, 136-143.

[33] Chang, T., and Gao, H. (2003). Size Dependent Elastic Properties of a Single-Walled Carbon Nanotube via a Molecular Mechanics Model. J. Mech. Phys. Solids 51, 10591074.

[34] Li, C., and Chou T. W. (2003). A Structural Mechanics Approach for the Analysis of Carbon Nanotubes. International Journal of Solids and Structures 40, 2487-2499.

[35] Fan, C. W., Liu, Y. Y., and Hwu, C. (2009). Finite Element Simulation for Estimating the Mechanical Properties of MultiWalled Carbon Nanotubes. Appl. Phys. A 95, 819-831.

[36] Tserpes, K. I., and Papanikos, P. (2005). Finite Element Modeling of Single-Walled Carbon Nanotubes. Composite 36: part B, 468-477.

[37] Fan, C. W., Huang, J. H., Hwu, C., and Liu, Y. Y. (2008). Mechanical Properties of Single-Walled Carbon Nanotubes-a Finite Element Approach. Adv. Mater. Res., 937-942

[38] Cornell, W. D., and et al. (1995). A Second Generation Force Field for the Simulation of Proteins, Nucleic Acids, and Organic Molecules. J. Am. Chem. Soc., 117 (19), 5179-5197.

[39] Jorgensen, W. L., and Severance, D. L. (1990). AromaticAromatic Interactions: Free Energy Profiles for the Benzene Dimer in Water, Chloroform, and Liquid Benzene. J. Am. Chem. Soc., 112 (12), 4768-4774.

[40] Natsuki, T., Tantrakarn, K., and Endo, M. (2004). Prediction of Elastic Properties for Single-Walled Carbon Nanotubes. Carbon 42, 39-45.

[41] Mohammad P. E., and Awang, M. (2012). A Finite Element Model to Investigate the Stress-Strain Behavior of Single Walled Carbon Nanotube. Advanced Structured Materials 16, 369-381.

[42] Giannopoulos, G. I., Kakavas, P. A., and Anifantis, N. K. (2008). Evaluation of the Effective Mechanical Properties of Single Walled Carbon Nanotubes Using a Spring Based Finite Element Approach. Computational Materials Science 41, 561569.

[43] Lier, G. V., Alsenoy, C. V., Doren, V. V., and Geerlings P. (2002). Ab Initio Study of the Elastic Properties of SingleWalled Carbon Nanotubes and Graphene. Chemical Physics Letters 326, 181-185.

[44] Shokrieh, M. M., and Rafiee, R. (2010). Prediction of Young's Modulus of Graphene Sheets and Carbon Nanotubes Using Nanoscale Continuum Mechanics Approach. Materials and Design 31, 790-795.

[45] Lu, J. P. (1997). Elastic Properties of Carbon Nanotubes and Nanoropes. Physical Review Letters, Vol. 79, 1297-1300.

[46] Jin, Y., and Yuan, F. G. (2003). Simulation of Elastic Properties of Single-Wwalled Carbon Nanotubes. Composites Science and Technology 63, 1507-1515.

[47] Selmi, A., Friebel, C., Doghri, I., and Hassis, H. (2007). Prediction of the Elastic Properties of Single Walled Carbon Nanotube Reinforced Polymers: A Comparative Study of Several Micromechanical Models. Composites Sci. Technol. 67, 2071-2084. 
[48] Mohammad, P. E., In Numerical And Experimental Evaluation Of Carbon Nanotube/Polypropylene Composites Using Nonlinear Finite Element Modeling, Ph. D. thesis, University Teknologi Petronas, 2013.

[49] Kalamkarov, A. L., Georgiades, A. V., Rokkam, S. K., Veedu, V. P., and Ghasemi-Nejhad, M. N. (2006). Analytical and Numerical Techniques to Predict Carbon Nanotubes Properties. Int. J. Solids Struct. 43 (22), 6832-6854.
[50] Lee, J. H., and Lee, B. S. (2012). Modal Analysis of Carbon Nanotubes and Nanocones Using FEM. Comput. Mater. Sci. $51(1), 30-42$.

[51] Yakobson, B. I., Brabec, C. J., and Bernholc J. (1996). Structural Mechanics of Carbon Nanotubes: From Continuum Elasticity to Atomistic Fracture. J. Comput. Aided Mater. Des. $3,173-182$. 